\title{
Note sur les femmes et la féminisation de l'armée dans quelques revues d'histoire militaire
}

\section{Vincent Joly}

\section{(2) OpenEdition}

1 Journals

\section{Édition électronique}

URL : https://journals.openedition.org/clio/1402

DOI : $10.4000 /$ clio. 1402

ISSN : 1777-5299

Éditeur

Belin

Édition imprimée

Date de publication : 1 novembre 2004

Pagination : 135-145

ISBN : 2-85816-755-9

ISSN : 1252-7017

Référence électronique

Vincent Joly, « Note sur les femmes et la féminisation de l'armée dans quelques revues d'histoire militaire », Clio. Histoire, femmes et sociétés [En ligne], 20 | 2004, mis en ligne le 01 janvier 2007, consulté le 23 avril 2022. URL : http://journals.openedition.org/clio/1402 ; DOI : https://doi.org/ 10.4000/clio.1402

Ce document a été généré automatiquement le 23 avril 2022.

Tous droits réservés 


\title{
Note sur les femmes et la féminisation de l'armée dans quelques revues d'histoire militaire
}

\author{
Vincent Joly
}

1 Au début des opérations en Irak, à la fin du mois de mars 2003, le soldat Jessica Lynch de la $507^{\mathrm{ème}}$ compagnie de maintenance fut capturé par les Irakiens avant d'être libéré quelques jours plus tard par les forces spéciales américaines. Rapidement, grâce au zèle du service de presse du commandement puis des médias eux-mêmes, cette jeune femme devint une figure emblématique du patriotisme mais révéla aussi l'importance de la présence féminine dans l'armée des États-Unis ${ }^{1}$. Quelques jours après le début de la guerre en Irak, le New York Times consacrait un article à cette réalité nouvelle intitulé » A new role for women as a new war unfolds $»^{2}$.

2 En octobre 1975, le président G. Ford signait une loi autorisant les femmes à entrer dans les académies militaires fédérales. Dès la rentrée 1976, une soixantaine de candidates furent intégrées à West Point ${ }^{3}$. Elles en sortirent en 1980, après avoir accompli les quatre années du cursus de cet établissement. Ces jeunes femmes ne se considéraient pas comme des militantes des droits des femmes, mais partageaient les mêmes motivations que leurs collègues masculins. Elles eurent à affronter une hostilité réelle de la part de ces derniers pour lesquels l'intégration des femmes dans l'institution la plus prestigieuse des forces armées américaines était plutôt mal vécue ${ }^{4}$. Cette décision correspondait au tournant que prenaient alors les forces armées américaines en raison de l'abandon de la conscription, décidé en 1973, et de la fin de la guerre au Vietnam. Depuis, les femmes ont été nombreuses à chercher à s'engager, pouvant désormais accéder, au moins théoriquement, à tous les grades et à toutes les armes.

3 La nouvelle période qui s'ouvre alors tranche par rapport aux décennies qui ont suivi la Deuxième Guerre mondiale. En effet, entre 1941 et 1945, environ 350000 femmes ont revêtu l'uniforme de l'armée américaine. Jamais plus de 120000 d'entre elles se sont trouvées en même temps sous les drapeaux, ce qui représentait un peu plus de $2 \%$ des effectifs totaux. Aucune n'a servi dans des unités de combat. Cependant, 88 d'entre elles 
ont été capturées par les Japonais au moment de la conquête des Philippines, la plupart appartenaient aux formations médicales. En effet, il n'y eut pas pendant la guerre de pression pour leur ouvrir les emplois autres que ceux qu'elles occupaient dans les bureaux, la santé, etc. de la part même des responsables féminines. Ainsi, celles-ci craignaient qu'en leur confiant des missions habituellement dévolues aux hommes, on mette en doute leur féminité 517000 servirent hors des États-Unis, dont les deux tiers en Europe.

4 Cette proportion est largement inférieure à celle des forces armées britanniques qui comptent $8,2 \%$ de femmes à la fin de la Deuxième Guerre mondiale 6 . Celles qui servaient dans l'Auxiliary Territorial Service pouvaient être affectées à la défense antiaérienne où leur nombre en 1944 était supérieur à celui des hommes et où leurs compétences étaient largement reconnues. En revanche, et contrairement à une idée reçue, elle est tout à fait comparable à celle de l'armée soviétique dans laquelle toutefois, des femmes pilotes, constituant trois régiments entièrement féminins, participèrent aux combats aériens ${ }^{7}$.

$5 \mathrm{Au}$ lendemain de la guerre, l'armée américaine n'attire plus les femmes malgré la création d'un organisme spécialisé qui devait favoriser leur entrée ou leur maintien dans les institutions militaires. Une loi votée au début de la guerre froide autorise leur engagement permanent mais, d'une part, elle limite leur nombre à $2 \%$ de l'effectif total des armées et, d'autre part, elle les exclut des grades supérieurs à celui de colonel. En outre, elle maintient leur exclusion des combats et même du service à la mer sauf sur les navires de transport et les navires hôpitaux ${ }^{8}$. Ainsi, jusqu'au début des années soixante-dix, leur nombre a stagné et même régressé, notamment après la guerre de Corée, tombant à $1,5 \%$ des effectifs totaux en 1968. Pendant la guerre du Vietnam, cette proportion n'évolue guère en dépit d'un assouplissement de la loi qui leur ouvre désormais tous les grades et supprime le numerus clausus ${ }^{9}$. Le contraste est donc frappant entre ces années et la décennie suivante au cours de laquelle on atteint les $8 \%$, puis les $15 \%$ à la veille de la dernière guerre en Irak ${ }^{10}$. Encore ce chiffre global masquet-il une forte inégalité par arme. Si l'on ne compte que $6 \%$ de femmes dans les « Marines », elles représentent en revanche 19\% des effectifs de l'Air Force.

6 Il y a dans ce phénomène une véritable révolution dont témoigne une bibliographie importante, mêlant toutes les approches. Les quelques observations qui suivent visent simplement à s'interroger sur la place qu'occupent comme thèmes de recherche les femmes et la féminisation de l'armée dans quelques revues d'histoire militaire plus particulièrement américaines.

7 Aux États-Unis et en Grande-Bretagne, l'histoire militaire occupe une place beaucoup plus importante qu'en France. Elle bénéficie d'un grand nombre de revues de qualité qui abordent tous les problèmes qui touchent aux questions militaires ${ }^{11}$. En outre, chaque arme, terre, air, mer et "marines", dispose de sa revue d'histoire dans lesquelles on trouve souvent de remarquables travaux. On peut y associer d'autres revues qui ne sont pas directement consacrées à l'histoire mais plutôt à la sociologie, aux études stratégiques ou autres et qui sont aussi largement ouvertes aux historiens. C'est le cas notamment de Armed Forces and Society qui aborde depuis bientôt une vingtaine d'années, plutôt sous l'angle de la sociologie, la question de la féminisation des armées comme celle de l'intégration des minorités. On pourrait y ajouter le Journal of Strategic Studies, Small Wars and Insurgencies, Comparative Strategy, etc..., autant de revues qui font parfois une place aux historiens ${ }^{12}$. Avec le Military History Quarterly, le 
public anglophone dispose en outre d'une revue plus accessible qui n'est pas prioritairement destinée aux universitaires, mais qui offre des articles de haut niveau et une iconographie particulièrement soignée.

8 Aux États-Unis, la revue d'histoire militaire de référence est le Journal of Military History, qui a pris la suite de Military Affairs en 1991. Chaque livraison contient cinq ou six articles sur des sujets très variés couvrant toutes les époques, tous les continents et une grande variété de thématiques. En outre, elle offre fréquemment des synthèses historiographiques importantes. L'un des intérêts majeurs de cette revue réside dans sa très abondante rubrique consacrée aux comptes-rendus d'ouvrages. En outre, tous les ans, elle propose une liste des travaux universitaires intéressant l'histoire militaire présentés en Grande-Bretagne, au Canada et, bien sûr, aux États-Unis. C'est sur cette revue que s'appuient les quelques observations qui suivent.

On est frappé par l'abondance de la littérature consacrée à la guerre et au genre en français et en anglais, ainsi que par le dynamisme qu'elle manifeste depuis quelques années ${ }^{13}$. On a un tableau tout à fait différent lorsqu'on se penche sur les revues d'histoire militaire. J'ai choisi, de manière tout à fait arbitraire, le Journal of Military History mais aussi une revue plus récente, publiée en Angleterre et dirigée par Hew Strachan et Dennis Showalter, War in History. Le dépouillement concerne non seulement les articles mais aussi les bibliographies, les informations les plus diverses comme les annonces de colloques, de congrès, les listes de thèses et les annonces publicitaires des maisons d'édition entre 1996 et 2001.

Entre 1996 et 2002 (du volume 3 au volume 9, soit 28 numéros), War in History a publié deux articles sur le thème des femmes et/ou de la féminisation dans les armées. Aucun ne traite du cas américain. Le plus long s'intéresse à un sujet tout à fait neuf, en tout cas pour le profane, sur les femmes combattantes au Japon au début de l'ère Meiji ${ }^{14}$. Le second est dû à M. van Creveld, qui est professeur à l'Université Hébraïque de Jérusalem et grand spécialiste d'histoire militaire" ${ }^{15}$. Intitulé "Armed but not dangerous : women in the Israeli forces ${ }^{16}$, son propos dépasse le cas de la seule armée israélienne. Il estime en effet, et il reprend cette thèse dans Les Femmes et la Guerre, que la présence des femmes dans l'armée dévalue l'institution et contribue à son déclin sans apporter de compétence ou de savoir-faire supplémentaires. Dans son article, il rappelle que, contrairement à l'image traditionnelle, les femmes sont exclues des unités combattantes de Tsahal depuis décembre 1947, après qu'une unité mixte du PALMACH (compagnie d'assaut de la Hagana) a été massacrée et ses membres mutilés. Van Creveld souligne ainsi que cette armée ne peut servir de modèle pour l'intégration des femmes dans l'institution militaire. Il estime, en outre, que le déclin des Forces de Défense Israéliennes (IDF), qui aurait commencé en 1982 avec l'opération Paix en Galilée au Liban et se serait aggravée avec la première Intifada, s'accompagne d'une féminisation accrue. Celle-ci découragerait les hommes de s'engager et dévaluerait le prestige de l'uniforme dans la société. De plus, les femmes, qui reçoivent désormais une instruction de combat, ne sont pas présentes aux premières lignes et ont accès à tous les grades sans en avoir nécessairement les compétences. En fait, on retrouve ici les arguments traditionnels des opposants à une stricte égalité entre hommes et femmes au sein des armées quelles qu'elles soient.

11 Cet article polémique lui a valu une réponse, hélas très brève, d'E. Kiesling, un an plus tard. L'historienne américaine, spécialiste de la doctrine militaire française de l'entredeux-guerres et qui enseigne à l'US Army War College, reproche à van Creveld de poser 
le problème en termes plus idéologiques qu'historiques. Ainsi, elle s'interroge sur la pertinence de la comparaison avec l'expérience américaine au Vietnam, soulignant au passage que les difficultés rencontrées alors par l'armée américaine ne devaient pas grand-chose à une féminisation de l'institution. En bref, selon elle, la thèse de van Creveld nous renseignerait davantage sur les attitudes masculines des Israéliens que sur les femmes de Tsahal. ${ }^{17}$.

Les recensions témoignent du même faible intérêt que suscite ce thème. En 28 livraisons, deux ouvrages seulement le concernent. Le premier est celui de L. D. Meyer qui pose la question de la compatibilité entre féminité et statut militaire en s'appuyant sur une étude approfondie du Women's Army Corps (WAC) et de son chef, le colonel Oveta Hobby, pendant la Deuxième Guerre mondiale ${ }^{18}$. Le second appartient à la veine inépuisable des livres sur les amazones du Dahomey sans y apporter de révélations particulièrement remarquables ${ }^{19}$.Dans les deux cas, les recensions, tout en étant critiques, sont largement positives. D. Ulbrich, cependant, interroge l'idée, centrale, de Leisa Meyer selon laquelle l'idéal masculin du bon soldat de l'armée américaine correspond à celui du bon adulte mâle dans la société en général. Selon lui, cette idée ne prend sans doute pas assez en compte l'environnement militaire qui fait du jeune soldat un automate alors que le jeune civil demeure autonome.

Le Journal of Military History confirme le caractère secondaire du thème des femmes et de la féminisation des armées. Entre 1999 et janvier 2004 (du volume 63 à la première livraison du volume 68) aucun article ne lui est consacré. Cependant, les copieuses recensions que contiennent chacun des quatre numéros annuels nuancent cette première impression et témoignent du dynamisme relatif des recherches dans ce domaine. En effet, dans tous ou presque, figure le compte-rendu d'un ou de plusieurs ouvrages qui touchent à ce thème. En tout, 22 livres sont ainsi analysés, traitant aussi bien des infirmières que des aviatrices ou encore des femmes de l'US Navy ${ }^{20}$. Parmi eux, il faut faire une place particulière à Amazons to fighter Pilots: A Biographical Dictionary of military Women dont R. L. Janda n'hésite pas à écrire qu'il s'agit d'une référence désormais incontournable pour tous les chercheurs qui s'intéressent aux femmes et à la guerre $^{21}$.

Les questions contemporaines ne sont pas négligées et plusieurs recensions concernent des travaux abordant la question de la féminisation des armées actuellement. Il faut notamment citer le débat contradictoire entre L. M. Fenner et M. de Young dans Women in Combat...22. Eugenia Kiesling estime que le temps des débats sur cette question devrait être dépassé car, écrit-elle:» Women being integral to virtually every aspects of military life, it is time to stop arguing and to divert our efforts instead into making the system work. ${ }^{23}$ On peut sans doute nuancer cette opinion en observant la persistance des controverses autour du harcèlement sexuel dans les forces armées américaines. Ainsi, pour ne prendre qu'un exemple, l'US Navy a été éclaboussée en 1991 par un scandale dont deux amiraux firent les frais, à la suite d'une soirée d'intégration des femmes-pilotes. D'ailleurs, des études récentes montrent que les pratiques militaires quotidiennes peuvent excuser voire même encourager encore ce genre de pratique et/ ou de discrimination à l'égard des femmes ${ }^{24}$.

Le JMH propose encore deux rubriques essentielles qui permettent de mesurer le dynamisme des thèmes de recherche. La première est le dépouillement systématique d'environ 400 périodiques qui offre ainsi un panorama quasi complet des principales publications anglophones qui s'intéressent à l'histoire militaire de près ou de loin. Les 
femmes et la féminisation des armées ne sont l'objet que d'une vingtaine de références, parmi lesquelles Minerva est la revue la plus citée. Ces chiffres sont faibles et ce constat est confirmé par l'absence de toute renvoi sur ce thème dans 6 livraisons du JMH.

La seconde rubrique est très importante car elle relève une fois par an, dans le numéro 3 publié en juillet, les thèses soutenues aux États-Unis, au Canada et au Royaume-Uni touchant à l'histoire militaire, mais dans toutes les disciplines, en histoire, en philosophie, en journalisme, en science politique et dans le cadre des Women's Studies. Sur environ 1300 thèses recensées, soit une moyenne de 260 par ans, 25 portent sur les femmes et la féminisation des armées du monde entier. Sans surprise, les études par arme, Marine, Aviation, Santé, etc. dominent très largement et deux d'entre elles seulement proviennent des Women'Studies. On note au passage que le cas des femmes d'origine afro-américaine semble très peu étudiée 25 .

Dans son excellente Histoire de la guerre, John Keegan écrit que « La guerre est la seule activité humaine vis-à-vis de laquelle les femmes, à d'infimes exceptions près, ont préféré toujours et partout garder leurs distances ${ }^{26}$. Certes, cette affirmation est tout à fait contestable, mais elle semble confirmée par la lecture des revues d'histoire militaire. Il ne s'agit pas d'un monopole d'historiens. J. Goldstein indique que l'influente revue Foreign Affairs n'a consacré aucun article sur la question du genre entre 1990 et $1996^{27}$. Il serait sans doute excessif d'inférer de ces constats qu'il existe une réelle volonté d'ostracisme. En effet, les ouvrages les plus importants font l'objet d'analyses critiques toujours de grande qualité et leur contribution est reconnue dans les sociétés d'histoire militaire. Ainsi, l'ouvrage de Kathleen Broome Williams sur le rôle des femmes scientifiques dans la marine américaine pendant la Deuxième Guerre mondiale a été justement récompensé par la North American Society for Oceanic History ${ }^{28}$.

Plus simplement, force est de constater que le thème est encore marginal et que, même dans l'opinion publique, la stricte égalité entre hommes et femmes dans l'armée est loin de faire l'unanimité. Un sondage Gallup cité par The New York Times du 7 avril 2003 indique qu'une très courte majorité d'Américains est favorable à la présence des femmes dans les unités de combat. La question suscite davantage d'intérêt chez les politistes et les sociologues que chez les historiens et touche souvent au problème de la cohésion des groupes restreints que forment en opération les combattants ${ }^{29}$. Il y a là peut-être aussi la marque d'une histoire militaire qui, certes, se renouvelle depuis plus d'une décennie mais qui est encore peu faite par des historiennes et qui privilégie la compréhension de la bataille au sens le plus large ${ }^{30}$.

\section{BIBLIOGRAPHIE}

BRAGG R., 2003, I am a soldier, too : The Jessica Lynch story, New York, A. Knopf.

BRODIE L.F., 2000, Breaking out : VMI and the coming of Women, New York, Praeger.

BRoome Williams K., 2001, Improbable Warriors: Women Scientists and the US Navy in World War Two, Annapolis Naval Institute Press. 
JANDA L..R, 1998, Stronger than custom : West Point and the admission of Women, University of Oklahoma.

CAPDEVILA L., ROUQuet F., Virgili F., VoldMAn D., 2003, Hommes et femmes dans la France en guerre (1914-1945), Paris, Payot.

CReVeld M. Van, 1977, Supplying War. Logistics from Wallenstein to Patton, Cambridge, Cambridge University Press.

-, 1998, Tsahal. Histoire critique de la Force Israélienne de Défense, Paris, Éditions du Rocher.

-, 2002, Les Femmes et la Guerre, Paris, Éditions du Rocher.

EDGERTON R.B., 2000, Warrior Women : The Amazons of the Dahomey and the nature of War, Boulder, West View Press.

FENNER L.M., Young M. de, 2001, Women in Combat : Civic duty or military liability ?, Washington, Geogetown University Press.

GOLDSTEIN J.S., 2001, War and Gender. How gender shapes the war system and vice versa, Cambridge, Cambridge University Press.

Goodson S., 2002, Serving Proudly: A History of Women in the US Navy, Annapolis, Naval Institute Press.

HoLm J., 1992, Women in the Military : An unfinished Revolution, Novato, Presidio Press (édition révisée).

JACKSON K., 2000, They called them Angels : American military Nurses in World War Two, Westport, Praeger.

JANDA L., 1998, Stronger than Custom: West Point and the admission of Women, University of Oklahoma.

KATZENSTEIN M.F., REPPY J., eds., 1999, Beyond zero tolerance : Discrimination in military culture, Lanham, Rowman and Littlefeld.

KeEGAN J., 1993, Anatomie de la bataille, Paris, Robert Laffont.

-, 1996, Histoire de la guerre. Du néolithique à la guerre du Golfe, Paris, Editions Dagorno.

KIESLING E., 1996a, « If it ain't broke, don't fix it : French military doctrine between the world wars », War in History, vol. 3, n² 2, p. 208-224.

-, 1996B, Arming against Hitler : France and the limits of military planning, University Press of Kansas.

LANGLEY W., 2002, Flying higher : The Women Airforce Service Pilots of World War Two, North Haven, Linnet Books.

MOORE B., 1996, To Serve my Country, to Serve my Race, New York, New York University Press.

MEYER L., 1996, Creating GI Jane : Sexuality and Power in the Women's Army Corps in World War Two, New York, Columbia University Press.

PENNIngton R. , 2002, Wings, Women \& War : Soviet airwomen in World War Two Combat, Lawrence, University Press of Kansas.

-, HighAm R., 2003, Amazons to fighter pilots : A Biographical Dictionary of military Women, Westport, Greenwood Press, 2 volumes.

WRIGHT D.E., 2001, « Female combattants and Japan's Meiji restoration : the case of Aizu », War in History, vol. 8, $\mathrm{n}^{\circ} 4$, p. 396-417. 


\section{NOTES}

1. En fait, comme le révéla la presse par la suite, la libération de Jessica Lynch fut largement « fabriquée » à des fins de propagande. Au demeurant, elle donna lieu à une abondante production d'articles et aux souvenirs de l'intéressée elle-même, recueillis par un journaliste (Bragg 2003).

2. The New York Times, Sunday, April 6, 2003.

3. Sur l'admission des femmes à West Point : Janda 1998.

4. Janda 1998. Cependant, toutes les institutions ne se sont pas ouvertes au même moment. Ainsi, l'école militaire préparatoire Virginia Military Institute, n'accepte les femmes que depuis l'été 1997 : Brodie 2000.

5. Meyer 1996, citée par Van Creveld $2002: 157$.

6. La proportion maximum de femmes dans l'armée britannique, toutes armes confondues, atteint 9,3\% en septembre 1943.

7. Cette question a fait l'objet d'une littérature relativement abondante dont, parmi les dernières publications : Pennington 2002. Rappelons que c'est en Finlande pendant la Deuxième Guerre mondiale que l'armée compta la plus forte proportion de femmes, les «Lottas », qui représentaient le quart des effectifs masculins mais dont aucune n'appartenait à des formations de combat.

8. Holm 1992.

9. Sur ce théâtre d'opération, les femmes servent essentiellement dans les formations sanitaires. Huit d'entre elles ont été tuées.

10. Goldstein 2001: 93.

11. On trouvera une bonne présentation générale des nouvelles tendances en histoire militaire dans Les Cahiers du Centre d'études d'histoire de la Défense, $\mathrm{n}^{\circ}$ 2, 1997. La lettre du CEHD, Défense et Histoire, permet aux historiens de se tenir au courant des publications les plus récentes, y compris des articles publiés dans les principales revues étrangères. 12. Il existe une revue spécialisée, mais que je n'ai jamais lue, intitulée Minerva. Quaterly Report on Women and the Military. Les sommaires que j'ai pu consulter indiquent de nombreux articles sur le thème de la féminisation, apparemment plus dans la veine de la « gender history » que dans celle de l'histoire militaire proprement dite. Elle touche aux questions très contemporaines. À titre indicatif, dans la livraison de l'hiver 2001, on trouve un article consacré aux femmes dans les unités néerlandaises de maintien de la paix.

13. Parmi de nombreuses références : Capdevila, Rouquet, Virgili, Voldman 2003. Précieuse bibliographie, p.325-341.

14. Wright 2001: 396-417.

15. M. Van Creveld est l'auteur, entre autres, d'une remarquable étude sur l'histoire de la logistique (1977), d'une stimulante histoire de l'armée israélienne (1998), mais aussi d'un essai plus polémique sur les femmes et la guerre (2002).

16. Vol. 7, n ${ }^{\circ} 1$, janvier 2000, p. 82-98.

17. Eugenia Kiesling est l'auteur d'un excellent article dans War in History (Kiesling 1996a). Elle a publié Arming against Hitler... : Kiesling $1996 \mathrm{~b}$.

18. Meyer 1996. Compte-rendu par D. Ulbrich dans le $n^{\circ} 1$, vol. 7, 2000, p. 118-120.

19. Edgerton 2000. Compte-rendu très complet par C.M. Peniston-Bird dans le $\mathrm{n}^{\circ} 4$, vol. 8, 2001, p. 483-485.

20. Quelques exemples : Jackson 2000, dans le $n^{\circ} 1$, vol. 65, janvier 2001, p. 227, par C.J. Moore ; Langley 2002, dans le n², vol. 67, avril 2003, p. 609, par Sh. Tucker. Dans la 
même livraison, p 608, R. L. Janda fait un excellent compte-rendu du livre de Goodson 2002 (ouvrage de référence avec une bibliographie de 38 pages).

21. Pennington Higham 2003. Compte-rendu par Robert Lance Janda dans $J M H, n^{\circ} 1$, vol. 68, janvier 2004.

22. Fenner Young 2001.

23. Vol. 66, $\mathrm{n}^{\circ} 2$, p. 649-650.

24. Comme le montrent les études rassemblées par Katzenstein Reppy 1999.

25. Sur cette question, $c f$. Moore 1996.

26. Keegan $1996: 108$.

27. Goldstein $2001: 34$.

28. Broome Williams 2001.

29. Cette question est aussi posée dans l'analyse de la présence des homosexuels dans l'armée.

30. Comme dans l'ouvrage pionnier de Keegan 1993 et, en France, dans les travaux de Stéphane Audoin-Rouzeau et d'Annette Becker.

\section{AUTEUR}

VINCENT JOLY

Vincent JOLY, professeur d'histoire contemporaine à l'Université Rennes 2, travaille sur l'histoire des colonisations et des décolonisations en Afrique. Il a notamment publié L'Europe et l'Afrique de 1914 aux années 1960, Rennes PUR, 1994. 